\title{
Commentary on social research about young people with congenital conditions
}

\author{
Priscilla Alderson \\ Social Science Research Unit, Institute of Education, University of London, UK
}

A s young people with serious congenital conditions live longer, research interest is growing in the quality of their everyday teenage and adult life. This commentary compares two general approaches to social research about these young people. The review is informed by my current research with young adults, who have cystic fibrosis, thalassaemia, sickle cell, spina bifida or Down's syndrome, about their views on the quality and value of their life. ('Prenatal Screening: Past, Present and Future', directed by Dr Ettorre, University of Helsinki, 1996-1999. funded by the European Commission Biomed II.)

A central topic of the research is the dilemma for young people: to balance being a careful patient responsible for keeping as healthy as possible, with being an ordinary person living a fulfilling social life, in what Jim in the paper by Tong et al calls 'the real world'. ' How do they reconcile these partly conflicting concerns of physical and social health? When the concerns conflict, which one matters more? Is their health a major preoccupation for them, or a relatively minor concern? Do they attribute their problems to their congenital condition or to other factors? Do they mainly identify themselves as a patient or a person?

The dilemma is also one for researchers. How do we gather and present fair, accurate accounts of young people's views on being mainly a patient or a person, an ill or a tolerably healthy individual? Their views are mixed and partly ambiguous; that is the nature of dilemmas. Their accounts may be affected by their current state of health, their confidence or shyness, passing moods of optimism or

Correspondence to: Dr Priscilla Alderson, Social Science Research Unit, Institute of Education, Unuversity of London, 18 Woburn Square, London WC1H ONS, UK. Fax: 01716126400

Manuscript accepted. 11 March 1998 pessimism, enjoyment of the interview or anxiety about it, and feelings that their views will be appreciated or criticised. Researchers have to convey the range and complexity of responses, and compress these into coherent reports.

The dilemma is repeated in professional and public opinion. Despite poor health, can individuals live fulfilled, highly valued lives? Is it kind or just to support lives of much suffering and frustration? Is it better, in certain severe cases, to withhold treatment after birth, and to terminate affected pregnancies? How essential and justifiable are costly medical and social interventions? Contrasting research approaches, emphasising either health or social concerns, and their advantages and limitations will now be reviewed.

\section{Health based social research}

The health based approach tends to involve the following. Protocols are designed by health professionals. Respondents are contacted through surgeries, hospital wards or clinics, and may be interviewed there. Their health records are consulted, and their own medical knowledge of their condition and compliance with prescribed treatment are checked. The interviewer is a health professional, and most of the questions mention the congenital condition. Researchers use such terms as: patients, disease, illness, problems, difficulties, limitations, worry and anxiety, restriction, function, control, management and strategies. The responses are reviewed in the light of psychological theories about normality, development, transition through life stages, and coping strategies. Originally a military term, 'strategies' extends the metaphor of life as a fight against disease. Treatment episodes and negative experiences and memories, even those from years earlier, tend to be emphasised. 
The advantages of the health based approach include easier access to large numbers of people, and high response rates. Hospital records provide a wide range of potential subjects, and overall can indicate the representativeness of the sample. Interviews are conducted more quickly and cheaply in a few treatment centres than by travelling to respondents' homes. Experienced health professionals conduct efficient interviews, which may be enhanced by the trust the respondents already have in the treatment centre and staff. Respondents may be helped with their problems, through having a sympathetic listener, and when research reports lead to improvements in their health care. Medical journal editors and clinicians are likely to take the findings seriously.

The disadvantages of the health based approach include the following. Interviewees may feel obliged to appear compliant and grateful, and preoccupied with their health care and morbidity. By default, social concerns like employment or personal relationships may be understated or omitted, and health concerns overstated; there are many reasons for failure to be confident, popular or an athletic star besides having a congenital defect. The defect may inadvertently be seen as the sole or primary influence; one example is later age on leaving the parental home, which is often more influenced by personal finances than health. When considered in isolation, people with a congenital condition easily seem disadvantaged, although their emotional and social problems and rewarding experiences may be widely shared by their nonaffected peers.

Psychological theories can distort rather than illuminate research conclusions. Evidence of adolescence, as a particular time of transition, non-compliance, and concern with self-esteem and peer relationships, under-estimates the transitions and concerns of other age groups. The few studies of adult patients' non-compliance show similarities with adolescents' behaviours. Differences may be more strongly linked to gender than to age, which also undermines the case for treating teenagers as a particularly vulnerable or volatile group. Assumptions about normality may be unrealistic.

\section{Socially based social research}

Researchers contact interviewees through informal networks, like self-help voluntary organisations, using opt-in methods. This is to respect their independence and to bypass their status as patients. They are interviewed at home or other place of their choice. Open questions enquire about family and friends, education and employment, likes and dislikes, and aspirations. Interviewees' relationships and activities are main themes, leaving them to introduce health issues as these become relevant during general narratives. The interview style is informal and the interviewer does not speak as an expert; when interviewees introduce medical terms they may be asked what these mean. The aim is to treat interviewees as experts in their own lives, and not to compare them against standards of normality or compliance. Health records are not checked, to respect privacy. Researchers use more neutral terms: people instead of patients; condition instead of disease; choice instead of strategy; treatment instead of management. Interviews range across many topics giving a sense of the interviewees as ordinary people who may see themselves as a friend and a sister, a musician and a mechanic, before being a patient.

The advantages are that this research gives a richer, more realistic view of the young people as contributing members of families and communities who have much in common with their peers. The effects of their health condition and treatments are seen more clearly within their daily life and their social, political and economic context, and the effects are seen in comparison with these other influences. Interviewees do not need to appear compliant or grateful to a social researcher. They may therefore be more honest and critical about their health care, its effectiveness, and their concern that it may eventually fail. This can encourage deeper reflections on the costs of life: dilemmas of balancing health with social interests when these conflict, the risks they think are worth taking, and the kinds of heroic treatment to improve the length or quality of life which they might accept. The disadvantages of socially based research include the following. It is harder to contact interviewees, and refusal rates tend to be high. Interviewees are likely to be unusually confident, and unrepresentative, though they may include severely impaired people. Without reviewing health records, it is harder to know what a representative sample would be like, and how it would compare with the interview group. The common finding that enjoyment and activity do not clearly correlate with clinically assessed health can only be checked against interviewees' reports about these assessments and their treatments. Interviewing and travelling take much longer. Morbidity and concerns about ill health and treatment may be under-reported. Selection occurs mainly after the data are collected, instead of before as when health based researchers choose narrower questions. This 
can make analysis and reporting even longer, more complicated processes. The results convey some of the complications of the 'real world' and the wide range of sometimes ambiguous fluctuating views, but they are not popular among medical journal editors who like clear, numerical summaries, and clinicians who prefer unambiguous easily applied conclusions. The research findings are therefore less likely to be publicised or to influence health and public policy.

\section{Summary}

Difficulty in finding the answers does not reduce the importance or urgency of the questions. As societies become more cost-conscious, and prenatal screening becomes more comprehensive, decisions are being made about which lives are worth supporting. Health professionals and the general public need to know more about the views of people with serious congenital conditions, including severely impaired people, and to involve them in finding informed answers to personal and social dilemmas.

Many young adults with serious congenital conditions are grateful for medical advances which enable them to live years, even decades, longer.
They are disheartened, as Tong's and other research ${ }^{1}$ shows, by social attitudes which still classify them as terminally ill patients, and not as potential friends, students, partners, employees or mortgage holders. These attitudes can prevent affected people from taking full advantage of the new opportunities created by modern health care. Compassionate attempts to increase health care support, such as by nurse counsellors, can, paradoxically, increase the images of vulnerable dependence and difference which reinforce social discrimination. These attempts turn public social problems into apparently private health ones; they treat the symptoms, like depression, but can exacerbate the disease of negative social attitudes. Young people I have interviewed would like health professionals to give essential health care, and also to help to encourage more positive public attitudes towards them. The range and complexity of people's responses is best understood through different and complementary research methods and studies.

\section{Reference}

1. Tong et al. Growing up with congentıal heart disease. Cardiology in the Young 1998; Vol 8 (3): 303-309 